\title{
Erratum to: Thymosin Alpha 1 is Associated with Improved Cellular Immunity and Reduced Infection Rate in Severe Acute Pancreatitis Patients in a Double-Blind Randomized Control Study
}

\author{
Xinying Wang, ${ }^{1}$ Weiqin Li, ${ }^{1}$ Chenglin Niu, ${ }^{1}$ Liya Pan, ${ }^{1}$ Ning $\mathrm{Li}^{1},{ }^{1}$ and $\mathrm{Jieshou} \mathrm{Li}^{1,2}$ \\ Erratum to: Inflammation \\ DOI 10.1007/s10753-010-9224-1
}

The original version of this article unfortunately contained two mistakes in the Tables 3 and 4. As shown in the Table 3, in the TA1 group, there were two patients with positive abdominal drainage culture, four patients with pneumonia, two patients with sepsis, whereas infection in 11 patients (eight patients with positive abdominal drainage culture, four patients with pneumonia, nine patients with sepsis) was observed in the control group. However, during the eight days after TA1 treatment, two patients with positive abdominal drainage culture and three patients with pneumonia were diagnosed while five patients with positive abdominal drainage culture, four patients with pneumonia, and four patients with sepsis were found. These differences in the positive rates of blood and abdominal drainage culture were statistically significant during the 28th follow-up period ( $P=0.012$ and $P=0.036$, Table 3 ). The captions and data in Tables 3 and 4 are in error and should be corrected as follows:

The online version of the original article can be found at http://dx.doi. org/10.1007/s10753-010-9224-1

\footnotetext{
${ }^{1}$ Research Institute of General Surgery, Jinling Hospital, Medical School of Nanjing University, 305 Zhongshan East Road, Nanjing, 210002, China

${ }^{2}$ To whom correspondence should be addressed at Research Institute of General Surgery, Jinling Hospital, Medical School of Nanjing University, 305 Zhongshan East Road, Nanjing, 210002, China. E-mail: njwangxinying@yahoo.com.cn
} 
Table 3. Comparison of Infection Events for the Two Study Groups After Treatment

\begin{tabular}{lcccccc}
\hline & \multicolumn{2}{c}{ AT group } & & \multicolumn{2}{c}{ CG group } \\
\cline { 2 - 3 } & Infected & Non-infected & & Infected & Non-infected & $P$ \\
\hline Blood(The 1st 8 days) & 0 & 12 & 4 & 8 & 0.093 \\
Blood(Within 28 days) & 2 & 10 & 9 & 3 & 0.012 \\
Sputum(The 1st 8 days) & 3 & 9 & 4 & 8 & 1 \\
Sputum(Within 28 days) & 4 & 8 & 5 & 7 & 0.371 \\
Abdominal cavity (The 1st 8 days) & 2 & 10 & 8 & 4 & 0.036 \\
Abdominal cavity(Within 28 days) & 2 & 10 & 4 & 8 \\
\hline
\end{tabular}

In all, six patients received abdominal drainage by needle puncture and four patients managed operation in the TA1 group. In the control group, ten patients received operation and two patients abdominal drainage. The difference of surgery rate was statistically significant between the two groups $(P=0.036$, Table 4$)$

Table 4. Comparison of the Treatment and Associated Events for the Two Study Groups After Randomization

\begin{tabular}{lcc}
\hline \multicolumn{1}{c}{ Variable } & AT group $(n=12)$ & CG group $(n=12)$ \\
\hline Duration of ICU stay, mean \pm SD, days & $24.6 \pm 19.6^{\mathrm{a}}$ & $50.5 \pm 25.7$ \\
Duration of hospital stay & $37.1 \pm 22.7^{\mathrm{b}}$ & $60.6 \pm 32.9$ \\
Operation, no. of patients & $4^{\mathrm{c}}$ & 10 \\
Only abdominal paracentesis, no. of patients & 6 & 2 \\
Conservative treatment, no. of patients & 2 & 0 \\
\hline
\end{tabular}

${ }^{a} P=0.014$ for comparison with $\mathrm{CG}$ group

${ }^{b} P=0.061$ for comparison with CG group

${ }^{c} P=0.036$ for comparison with CG group 